\title{
COMMENTARY
}

\section{Where does the lactate come from? A rare cause of reversible inhibition of mitochondrial respiration}

\author{
Bruno Levy*, Pierre Perez and Jessica Perny \\ See related research by Protti et al., http://ccforum.com/content/14/1/R22
}

\begin{abstract}
Biguanide poisoning is associated with lactic acidosis. The exact mechanism of biguanide-induced lactic acidosis is not well understood. In the previous issue of Critical Care, Protti and colleagues demonstrated that biguanide-induced lactic acidosis may be due in part to a reversible inhibition of mitochondrial respiration. Thus, in the absence of an antidote, increased drug elimination through dialysis is logical.
\end{abstract}

In the previous issue of Critical Care, Protti and colleagues presented a series of patients with severe hyperlactatemia secondary to biguanide intoxication [1]. Traditionally, hyperlactatemia in critically ill patients and particularly those in shock - was normally interpreted as a marker of secondary anaerobic metabolism due to inadequate oxygen supply inducing cellular distress [2]. This view has recently been challenged with the demonstration that, during shock states, lactate production is, at least in part, linked to an increased aerobic glycolysis through $\beta_{2}$ stimulation [3]. We recently demonstrated in a rat model that this mechanism occurs not only during sepsis (high or normal blood flow), but also during hemorrhagic shock (low blood flow) [4].

In clinical practice, there are clearly certain situations where hyperlactatemia is predominantly a reflection of tissue hypoperfusion with subsequent anaerobic metabolism. Shock states induced by low cardiac output should theoretically be accompanied by hypoxic hyperlactatemia. Cardiogenic shock, as demonstrated previously [5], is associated with hyperlactatemia with a very high lactate/pyruvate ratio. In theory, hemorrhagic shock should behave in an identical fashion. Nevertheless, hemorrhagic shock, when prolonged, becomes an inflammatory shock and may therefore behave as septic shock.

*Correspondence: b.levy@chu-nancy.fr

Service de Reanimation Médicale, CHU Nancy-Brabois, 54511 Vandoeuvre les Nancy, France
The problem encountered with sepsis is more complex, although at least two situations are usually accompanied with hypoxia-associated hyperlactatemia. The first situation is septic shock with catecholamine-resistant cardiocirculatory failure, especially in situations of low cardiac output. The second circumstance is septic shock pre-emptively observed prior to volumetric expansion, as illustrated in the study of Rivers and colleagues in which hyperlactatemia was associated with signs of poor oxygen delivery [6]. These two situations are nonetheless close to low-output states.

By definition, hypoxia blocks mitochondrial oxidative phosphorylation [7], thereby inhibiting ATP synthesis and reoxidation of NADH. This leads to a decrease in the ATP/ADP ratio and an increase in the NADH/NAD ratio. A decrease in the ATP/ADP ratio induces both an accumulation of pyruvate, which cannot be utilized by way of phosphofructokinase stimulation, and a decrease in pyruvate utilization by inhibiting pyruvate carboxylase, which converts pyruvate into oxaloacetate. An increased $\mathrm{NADH} / \mathrm{NAD}$ ratio also increases pyruvate by inhibiting pyruvate dehydrogenase, and hence its conversion into acetylcoenzyme A.

Consequently, the increase in lactate production in an anaerobic setting is the result of an accumulation of pyruvate that is converted into lactate, which stems from alterations in the redox potential. This conversion allows for the regeneration of some $\mathrm{NAD}^{+}$, enabling the production of ATP by anaerobic glycolysis - although the process is clearly less efficient from an energy standpoint (two ATP molecules produced versus 36). It is important to consider that the modification of the redox potential induced by an increase in the NADH/NAD ratio activates the transformation of pyruvate into lactate, and consequently increases the lactate/pyruvate ratio [8].

All in all, anaerobic energy metabolism is characterized by hyperlactatemia associated with an elevated lactate/ pyruvate ratio, greater glucose utilization and low energy production [9].

The exact mechanism of biguanide-induced lactic acidosis is not well understood. This infrequent complication is associated with high mortality. Biguanide drugs 
mainly exert their therapeutic effect by impairing hepatocyte mitochondrial respiration [10]. Recent observations have suggested that metformin, similarly to phenformin, may also inhibit mitochondrial respiration in tissues other than the liver [11].

In the previous issue of Critical Care, using indirect measurement of oxygen consumption, Protti and colleagues found that body oxygen consumption was markedly depressed despite a normal cardiac index evoking an inhibition of mitochondrial respiration [1]. Unfortunately, arterial lactate/pyruvate and acetoacetate/ $\beta$-hydroxybutyrate ratios, as reflections of cytoplasmic and mitochondrial redox states, were unavailable. Interestingly, there was a clear correlation between drug clearance, correction of lactic acidosis and normalization of oxygen consumption. Clearly, the inhibition of mitochondrial respiration is not the unique mechanism involved in biguanide-induced lactic acidosis, since pure inhibition of mitochondrial function during cyanide poisoning is associated with death in the absence of antidote [12], and, similarly, since lactic acidosis associated with the use of nucleoside analogue reverse transcriptase inhibitors is due to an impairment of mitochondrial oxidative phosphorylation and is also associated with high mortality despite prompt therapy [13].

To conclude, when looking at the literature, pure hypoxic causes of lactic acidosis are relatively rare in clinical practice. In the case of biguanide-induced lactic acidosis, the fact that the inhibition of mitochondrial respiration is reversible should encourage the early use of dialysis [14] in order to accelerate drug elimination.

\section{Abbreviations}

$\mathrm{NAD}$, nicotinamide adenine dinucleotid; $\mathrm{NADH}$, reduced form of NAD.

\section{Competing interests}

The authors declare that they have no competing interests.
Published: 1 April 2010

\section{References}

1. Protti A, Russo R, Tagliabue P, Vecchio S, Singer M, Rudiger A, Foti G, Rossi A, Mistraletti G, Gattinoni L: Oxygen consumption is depressed in patients with lactic acidosis due to biguanide intoxication. Crit Care 2010, 14:R22.

2. Bakker J, Jansen TC: Don't take vitals, take a lactate. Intensive Care Med 2007 33:1863-1865

3. Levy B, Gibot S, Franck P, Cravoisy A, Bollaert PE: Relation between muscle $\mathrm{Na}^{+} \mathrm{K}^{+}$ATPase activity and raised lactate concentrations in septic shock: a prospective study. Lancet 2005, 365:871-875.

4. Levy B, Desebbe O, Montemont C, Gibot S: Increased aerobic glycolysis through beta2 stimulation is a common mechanism involved in lactate formation during shock states. Shock 2008, 30:417-421.

5. Levy B, Sadoune LO, Gelot AM, Bollaert PE, Nabet P, Larcan A: Evolution of lactate/pyruvate and arterial ketone body ratios in the early course of catecholamine-treated septic shock. Crit Care Med 2000, 28:114-119.

6. Rivers E, Nguyen B, Havstad S, Ressler J, Muzzin A, Knoblich B, Peterson E, Tomlanovich M: Early goal-directed therapy in the treatment of severe sepsis and septic shock. N Engl J Med 2001, 345:1368-1377.

7. Alberti KG: The biochemical consequences of hypoxia. J Clin Pathol Suppl (R Coll Pathol) 1977, 11:14-20.

8. Leverve XM: Mitochondrial function and substrate availability. Crit Care Med 2007, 35(9 Suppl):S454-S460.

9. Levy B: Lactate and shock state: the metabolic view. Curr Opin Crit Care 2006, 12:315-321.

10. El-Mir MY, Nogueira V, Fontaine E, Averet N, Rigoulet M, Leverve X: Dimethylbiguanide inhibits cell respiration via an indirect effect targeted on the respiratory chain complex I. J Biol Chem 2000, 275:223-228.

11. Brunmair B, Staniek K, Gras F, Scharf N, Althaym A, Clara R, Roden M, Gnaiger E, Nohl H, WaldhäusI W, Fürnsinn C: Thiazolidinediones, like metformin, inhibit respiratory complex I: a common mechanism contributing to their antidiabetic actions? Diabetes 2004, 53:1052-1059.

12. Peddy SB, Rigby MR, Shaffner DH: Acute cyanide poisoning. Pediatr Crit Care Med 2006, 7:79-82.

13. Lewis W, Dalakas MC: Mitochondrial toxicity of antiviral drugs. Nat Med 1995, 1:417-422.

14. Peters N, Jay N, Barraud D, Cravoisy A, Nace L, Bollaert PE, Gibot S: Metforminassociated lactic acidosis in an intensive care unit. Crit Care 2008, 12:R149.

\section{doi:10.1186/cc8904}

Cite this article as: Levy B, et al.: Where does the lactate come from? A rare cause of reversible inhibition of mitochondrial respiration. Critical Care 2010, 14:136 